\section{Schwannoma retroperitoneal. Caso clínico}

\author{
CARLOS ÁLVAREZ Z. ${ }^{1,3}$, BELEN SANHUEZA P. ${ }^{1}$, \\ IVÁN RUIZ F. ${ }^{1, a}$, JORGE CASTILLO A. ${ }^{2}$

\section{Retroperitoneal schwannoma. Report of one case}

\begin{abstract}
Retroperitoneal schwannoma is an uncommon and usually benign condition. We report a 51-year-old woman presenting with a palpable pelvic tumor. The patient was operated on and during the intervention, two retroperitoneal tumors were found and resected. The postoperative course was favorable. Pathology confirmed the diagnosis of retroperitoneal benign shwannomas. After eight years of follow up, there is no evidence of malignant transformation.
\end{abstract}

(Rev Med Chile 2012; 140: 767-770).

Key words: General surgery; Neurilemmoma; Retroperitoneal neoplasms.

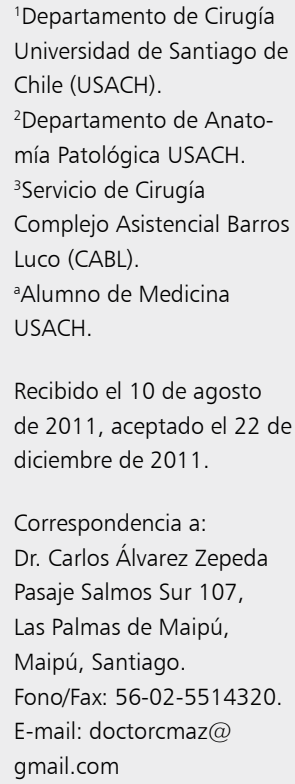

I os schwannomas o neurilemomas son tumores bien capsulados que se originan en las vainas de los nervios periféricos y que aparecen en jóvenes o adultos de edad media. Se presentan generalmente en la cabeza, el cuello, las extremidades y el mediastino posterior, siendo muy poco frecuentes en el retroperitoneo ${ }^{1}$. Constituyen el 0,5 a $2,7 \%$ de los tumores retroperitoneales ${ }^{2-5}$. La mayoría de los shwannomas son benignos y únicos, aunque hay descritos casos múltiples ${ }^{5,6}$. Se han informado casos malignos asociados a enfermedad de Von Recklinghausen, nevus melanocítico en niños y con hipercalcemia de origen paratiroi$\mathrm{deo}^{7-9}$. La mayoría de las veces su diagnóstico es un hallazgo en un paciente que se estudia por otra causa, o se presenta como un tumor sin diagnóstico preoperatorio, que sólo después de la cirugía y tras el estudio histológico se certifica su naturaleza ${ }^{6,7}$.

Comunicamos el caso de una paciente con un schwannoma retroperitoneal doble operado en el Servicio de Cirugía del Complejo Asistencial Barros Luco (CABL); se reporta su presentación clínica, imágenes preoperatorias, de la pieza quirúrgica y la histología, además del seguimiento de 8 años. Realizamos una revisión del tema, para lo cual se realizó una búsqueda a través del Catálogo Bello, LILACS, PubMed y SciELO sin restricción de tiempo o idioma.

\section{Caso clínico}

Paciente de sexo femenino de 51 años de edad, sin antecedentes mórbidos conocidos, que en noviembre de 2001 consultó a Servicio de Ginecología por síntomas climatéricos palpándose en forma incidental en el examen físico una masa pélvica. Esta fue descrita posteriormente en una ecografía transvaginal como sólida y ubicada en región pararetro-uterina izquierda de $65 \times 48 \times 49 \mathrm{~mm}$, con flujos Doppler positivos en su interior y separada del cuerpo uterino ocupando parcialmente el saco de Douglas, desplazando el útero a izquierda. Fue ingresada para cirugía con el diagnóstico de tumor sólido de anexo izquierdo. Se realizó por ginecólogo laparotomía exploradora, describiéndose útero y ovarios de aspecto normal, un quiste paraooforo derecho y una masa retroperitoneal pélvica, de aspecto sólido quística de unos $12 \mathrm{~cm}$ de diámetro, sin compromiso de órganos vecinos. Se decidió terminar la exploración y derivar a cirugía para tratamiento definitivo. Se completó estudio con tomografía computada (TC) de abdomen y pelvis (Figura 1), cistoscopia y colonoscopia normales y esofagogastroscopia que mostró una gastritis aguda congestiva y una hernia hiatal. Finalmente, fue intervenida en abril de 2003, encontrándose en región pélvica retroperitoneal 2 masas de $8 \times 8 \mathrm{~cm}$ 


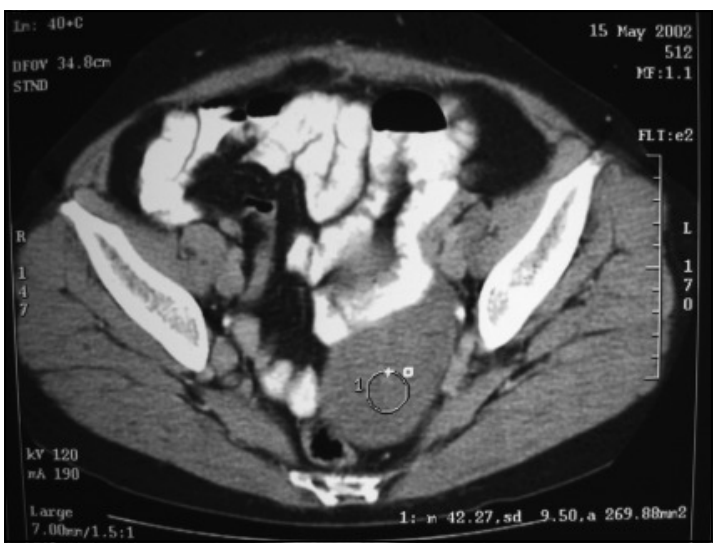

Figura 1. TC de abdomen y pelvis. Se observa la masa retroperitoneal de ubicación pélvica.

y $3 \times 4 \mathrm{~cm}$ (Figura 2), que se resecaron y enviaron a estudio, que fue informado posteriormente como 2 shwannomas retroperitoneales benignos (Figura 3). La paciente evolucionó favorablemente, siendo dada de alta al primer día pos operatorio, a 8 años de seguimiento la paciente se encuentra asintomática y sin evidencia de recidiva de la enfermedad.

\section{Discusión}

Los schwannomas son tumores originados de las células gliales periféricas que derivan del neuroectodermo. En 1910 Verocay fue el primero en reportar un caso al cual denominó neurofibroma ${ }^{10}$. En 1932 Masson dilucidó que tales tumores se originaban de las células de Schwann por lo que los denominó schwanomas ${ }^{11,12}$.

Los shwannomas pueden aparecer en varios órganos o en los troncos nerviosos de todo el cuerpo, excepto los pares craneanos I y II, que están desprovistos de células de Schwann. Corresponden aproximadamente al $8 \%$ de los tumores del cerebro, observándose frecuentemente en el VIII par $^{13}$. En los nervios espinales se desarrolla más frecuentemente a nivel lumbar. Es el tumor benigno más frecuente de la médula espinal, constituyendo el 20 a 30\% de los tumores de esta ubicación $\mathrm{n}^{5,13}$. En el retroperitoneo los shwannomas son raros, constituyendo aproximadamente el $1 \%$ de los tumores retroperitoneales ${ }^{14}$. Debido a que esta zona anatómica es flexible, a menos que el tumor alcance un tamaño considerable, estos

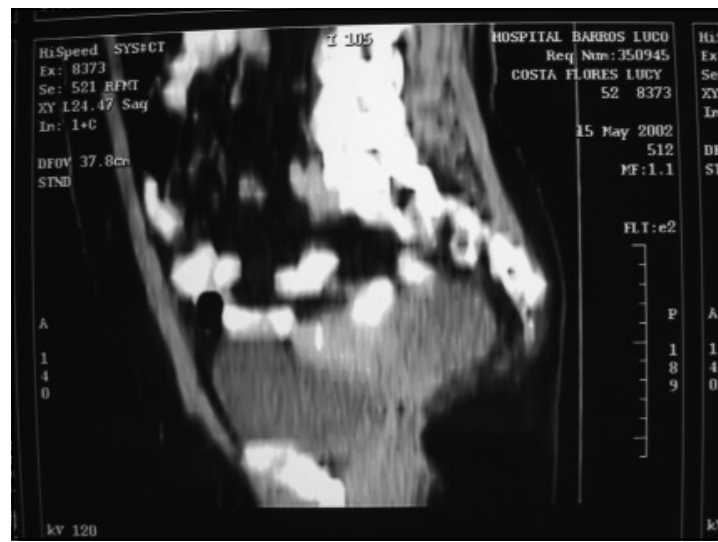

Figura 2. TC de abdomen y pelvis. Reconstrucción sagital. Se observa su ubicación retrouterina y el desplazamiento del órgano.

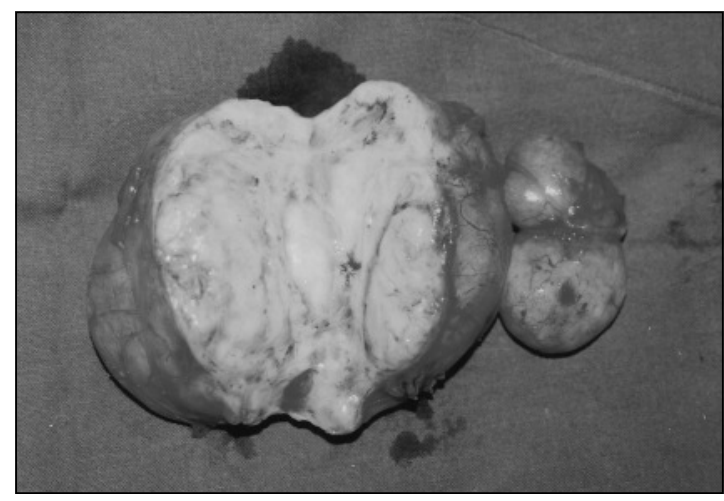

Figura 3. Pieza operatoria. La masa de mayor tamaño está escindida en forma longitudinal.

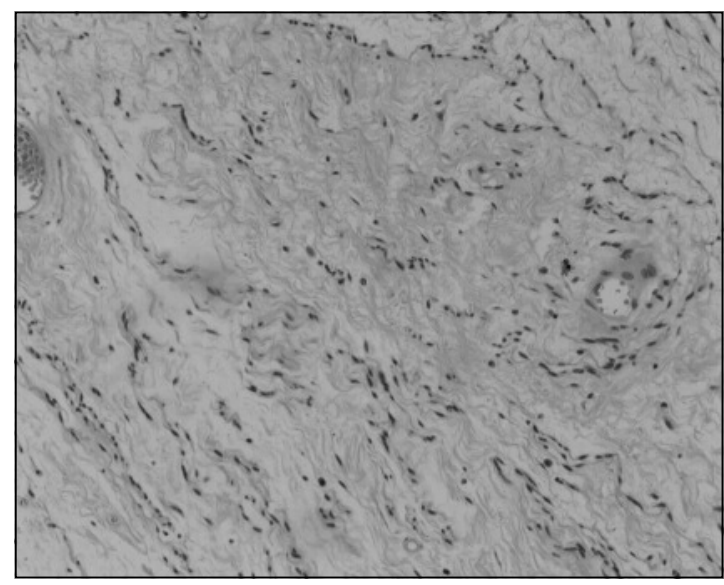

Figura 4. Histología: tinción HE x 10. Haces de células fusadas y onduladas con núcleos dispuestos en bandas. Corte con predominio de áreas Antoni B. 
tumores suelen ser asintomáticos y generalmente únicos ${ }^{5,10,15}$. Nuestro caso si bien fue asintomático presentó 2 masas retroperitoneales. Ya que la forma de presentación del schwannoma retroperitoneal es generalmente inespecífica y el diagnóstico es incidental, la confirmación preoperatoria es infrecuente, como sucedió en el caso por nosotros reportado. La edad del diagnóstico suele estar entre los $20 \mathrm{y}$ los 50 años. La incidencia observada es levemente más frecuente en las mujeres que en los hombres. Cuando presenta síntomas los más frecuentes son el dolor abdominal, la distensión abdominal y, la lumbalgia ${ }^{5,13}$. Otras veces, pero menos frecuentemente, se han reportado incontinencia urinaria o fecal, neuralgias o incluso déficit sensitivos ${ }^{10,13}$.

El diagnóstico inicial de tumor retroperitoneal se realiza gracias a las imágenes. Al igual que en nuestro caso muchas veces la primera aproximación es la ecografía, esta permite evaluar el tamaño, la ubicación y las características acústicas internas. Detecta la presencia de calcificaciones y cambios quísticos. Las lesiones típicas son heterogéneas, híper-ecoicas y contienen componentes quísticos prominentes, aunque estos signos no son específicos ${ }^{16,17}$. La tomografía computada es un examen más útil para diferenciar los tumores del retroperitoneo, en el shwannoma las imágenes más típicas muestran un tumor con márgenes lisos y nítidos con bordes reforzados, a menudo se puede encontrar licuefacción y necrosis y, puede haber signos de hemorragia intratumoral. Por esto se ha mencionado que es posible diferenciarlo de linfomas, hemangiomas, quistes y enfermedades del tejido conectivo $^{5}$. En la resonancia magnética (RM) el shwannoma se ve en $\mathrm{T} 1$ de igual intensidad que el músculo y en T2 se ve de intensidad mayor. La RM puede ser muy útil para ver el origen de la masa, su arquitectura vascular y el compromiso de otros órganos, incluida la médula espinal ${ }^{5,18}$.

Para el diagnóstico preoperatorio de estos tumores algunos autores han realizado biopsia por punción, guiada bajo TC o ecografía, la que es útil sólo si se consiguen suficientes células de Schwann en la muestra. Sin embargo, el pleomorfismo celular, el componente quístico y la gran vascularización no sólo no permiten el diagnóstico, sino que a veces hacen que el procedimiento ocasione hemorragia, infección o siembra de la masa, por esto es que muchos autores no la recomiendan ${ }^{7,18,19}$.

Histológicamente los schwannomas se clasifican en dos grupos. El Antoni tipo A: una zona celular compacta con un patrón en empalizada o en espiral; y un Antoni tipo B: una zona suelta, hipocelular y mixoide con espacios microquísticos que se componen de tejido mixoide y degenerado con menos células y sustancia gelatinosa. El sello de las variantes benignas es el patrón de áreas Antoni A alternadas con Antoni B, con intensa tinción de proteína $S 100^{5,13,18}$. La malignidad vendrá determinada por el número de mitosis, la invasión vascular o el pleomorfismo, no siendo el tamaño del tumor un factor determinante ${ }^{15}$.

El diagnóstico diferencial del schwannoma retroperitoneal incluye los quistes epiteliales, los abscesos, el neurofibrosarcoma (MPNST de su sigla en inglés, o tumor maligno de la vaina del nervio periférico), el meningioma sacro, ependimoma, cordoma, condrosarcoma, el tumor de células gigantes, el quiste óseo aneurismático, osteoblastoma, teratoma sacrococcígeo, lifoma y la transformación maligna de un tumor benigno ${ }^{13}$. Las lesiones no neoplásicas incluyen el pseudoquiste de páncreas, el linfocele y el urinoma. Aunque los hallazgos clínicos e imagenológicos pueden ser a veces similares entre estas lesiones, algunas características de la TC como la localización, el tamaño, la forma, las características de la pared, la presencia de tabiques o calcificaciones y el compromiso de estructuras adyacentes pueden sugerir en algunas ocasiones el diagnóstico específico ${ }^{20,21}$. También, como lo han reportado otros autores y como nuestro caso, el schwannoma en la cavidad pélvica podría diagnosticarse erróneamente como un tumor de ovario ${ }^{13,22}$.

Sin lugar a dudas el mejor manejo de los schwannomas es la resección completa, habitualmente por laparotomía, aunque se han publicado resecciones exitosas usando laparoscopias asistidas por minilaparotomías ${ }^{14,23}$. El sangrado es la complicación intraoperatoria más grave, cuando los vasos mayores están cerca del tumor. En nuestro caso se empleo la resección radical del tumor por laparotomía, asumiendo que nos enfrentábamos a una lesión neoplásica retroperitoneal de diagnóstico patológico desconocido, con el fin de asegurar el tratamiento óptimo y la sobrevida de nuestro paciente. Se han descrito como complicaciones de la cirugía, algunas molestias que están en relación con los nervios involucrados, como parestesia, debilidad y dolor lumbar y de extremidades ${ }^{18}$. Nuestra paciente no presentó molestias tras la cirugía ni en el seguimiento. Aunque los intentos 
de resección completa se han relacionado con más complicaciones, lo aconsejable es la total escisión del tumor con márgenes negativos, ya que se debe considerar la posibilidad de recurrencia en los shwannomas benignos resecados en forma parcial, sumado a demás a que no es posible excluir, ni con análisis de biopsias rápidas intraoperatorias la malignidad del tumor ${ }^{18,24}$. A 8 años de la operación nuestra paciente no presenta evidencias de recurrencia de la enfermedad.

\section{Conclusiones}

El schwannoma retroperitoneal es una enfermedad poco frecuente. Su forma de presentación es inespecífica y generalmente es un hallazgo. Puede confundirse con una neoplasia de ovario cuando es de ubicación pélvica. La TC y la RM son útiles para el enfrentamiento preoperatorio. No se recomienda la biopsia previa por punción. Su tratamiento es siempre la resección completa, en casos seleccionados puede ser operada por técnica mínimamente invasiva.

\section{Referencias}

1. Sewng Hyup Kim, Byung Ihn Choi. Retroperitoneal neurilemmoma: CT and MR findings. AJR 1992; 159: 1023-6.

2. Ueda M, Okamoto Y, Ueki M. A pelvic retroperitoneal schwannoma arising in the right paracolpium. Gynecol Oncol 1996; 60: 480-3.

3. Hayasaka K, Tanaka Y, Soeda S, et al. MR findings in primary retroperitoneal schwannoma. Acta Radiol 1999; 40: 78-82.

4. Regan JF, Juler GL, Schmutzer KJ. Retroperitoneal neurilemoma. Am J Surg 1977; 134: 140-5.

5. Qiang LI, Chuntao Gao, Juzi JT, Xishan Hao. Analysis of 82 cases of retroperitoneal schwannoma. ANZ J Surg 2007; 77: 237-40.

6. Hurley L, Smith JJ, Larsen CR, Silverman ML. Multiple retroperitoneal schwannomas: case report and review of literature. J Urol 1994; 151: 413-6.

7. De Diego Rodríguez E, Roca Edreira A, Martín García B, Hernández Rodríguez R, Portillo Martín JA, et al. Schwannoma benigno retroperitoneal. Aportación de un nuevo caso. Actas Urol Esp 2000; 24: 685-2.

8. Roth MJ, Medeiros LJ, Kapur S, Wexler LH, Mims S, Horowitz ME, et al. Malignant schwannoma with melanocytic and neuroepithelial diferentiation in an infant with congenital giant melanocityc nevus:a complex neurocristopathy. Human Path 1993; 24: 1371-5.3.
9. Mikami O, Matsuda T, Danno S, Komatz Y. A case of retroperitoneal schwannoma associated with primary hyperparathyroidism. Hinyokika Kiyo 1994; 40: 131-4.

10. Verocay J. Zur kenntnis der Neurofibrome. Beitr Pathol Anat Allg Pathol 1910; 48:1-5.

11. Masson P. Experimental and spontaneous schwannomas (peripheral gliomas): part I. Am J Pathol 1932; 8: 367-70.

12. Masson P. Experimental and spontaneous schwannomas (peripheralgliomas): part II. Am J Pathol 1932; 8: 389416.

13. Song JY, Kim SY, Park EG, Kim CJ, Kim Do G, Lee HK, et al. Schwannoma in the retroperitoneum. J Obstet Gynaecol Res 2007; 33 (3): 371-5.

14. Rocca JP, Amarillo H, Mihura M. Schwanoma retroperitoneal: un nuevo abordaje para su resección. Cir Esp 2003; 73 (5): 318-20.

15. Manuel JC, Alonso-Martín J, Fernández-Díaz MJ, Alonso-Gayon JL, Barcena-Barros JM, Corral-Mones J. Shwannoma benigno retroperitoneal. Cir Esp 2002; 72 (1): 50-2.

16. Do-Dai DD, Ho VB, Rovira MJ, Knight RW, Twomey PA. Retroperitoneal melanotic schwannoma: ultrasonographic features. J Clin Ultrasound 1995; 23: 42-8.

17. Takatera H, Takaha M, Takiuchi H, Namiki M, Ohnishi S, Sonoda T. Retroperitoneal schwannoma. Urology 1986; 28: 529-31.

18. Daneshmand S, Youssefzadeh D, Chamie K, Boswell W, Wu N, Stein JP, et al. Benign retroperitoneal schwannoma: a case series and review of the literature. Urology. 2003; 62 (6): 993-7.

19. Neifer R, Nguyen GK. Aspiration cytology of solitary schwannoma. Acta Cytol 1985; 29: 12-4.

20. Yang DM, Fung DH, Kim H, Kang FH, Kim SH, Kim FH, et al. Retroperitoneal Cystic Masses: CT, Clinical, and Pathologic Findings and Literature Review. RadioGraphics 2004; 24: 1353-65.

21. Álvarez ZC, Cerda CC, Jadue TA, Rojas RF, Abelleira PM, Hermansen TC, et al. Hematoma retroperitoneal espontáneo: Caso clínico. Rev Med Chile 2007; 135 (8): 1044-7.

22. Kentopp K, Jones MA, Decain M, Tarraza HM. Pelvic retroperitoneal schwannoma mimicking an ovarian neoplasm: report of a case and review of the literature. Eur J Gynecol Oncol 1998; 19: 57-9.

23. Theodosopoulos T, Stafyla VK, Tsiantoula P, Yiallourou A, Marinis A, Kondi-Pafitis A, et al. Special problems encountering surgical management of large retroperitoneal schwannomas. World J Surg Oncol 2008; 6: 107-12.

24. Carpenter W, Kernohan JW. Retroperitoneal ganglioneuromas and neurofibromas: a clinicopathological study. Cancer 1963; 16: 788-96. 